\title{
Efficient Construction of Quaternary Carbon: Stereocontrolled Synthesis of Novel Abacavir Analogue
}

\author{
Aihong Kim and Joon Hee Hong* \\ BK-2I Project Team, College of Pharmacy, Chosun University, Gwangin 501-759, Korea. E-mail: hongihiachoswacikr \\ Recened June 4. 2007
}

\begin{abstract}
This paper discusses the racemic and stereoselective synthetic route for novel 4 ' $\alpha$-methyl and 6 ' $\alpha$-methyl analogues of abacavir. The quaternary carbon at the 4'-position of carbocyclic nucleoside was installed successfully via a Claisen rearrangement. The stereocontrolled construction of a methyl group in the $6^{\prime} \alpha$ position was directed through the Felkin- $\Lambda$ nh rule. $\Lambda$ Bis-vinyl compound 9 was cyclized successfully using Grubbs ' catalyst II to provide a carbocycle nucleus for the target compound. The synthesized compound $\mathbf{1 5}$ showed moderate anti-] [IV activity $\left(\mathrm{EC}_{50}-10.67 \mu \mathrm{M}\right.$. MT-4 cell lines).
\end{abstract}

Key Words : Quaternary carbon, Abacavir, Claisen rearrangement, Ring-closing metathesis

\section{Introduction}

Eimerging drug-resistant virus strains and toxicity are major problems in antiviral chemotherapy, and a number of structurally modified nucleosides have been synthesized to overcome these drawbacks. More fundamental modifications to the pentofuranose moiety, such as carbocyclic nucleosides, have been reported to be compatible with their antiviral activity. Carbocyclic nucleosides are a group of compounds that are structurally similar to the natural nucleosides where the furanose oxygen is replaced by a methylene group. The replacement of the oxygen on the furanose ring by carbon is of particular interest because the resulting carbocyclic nucleosides have a greater metabolic stability to phosphorylase, ? which cleaves the glycosidic bond of nucleosides. Since the cyclopentane ring of the carbocyclic nucleosides can emulate the furanose moiety, a number of these compounds exhibit interesting biological activities, particularly in the areas of antiviral and anticancer chemotherapy. The recent discovery of olefinic carbocyclic nucleosides, such as abacavir $\mathbf{1}^{*}$ and entecavir, ${ }^{+}$as potential antiviral agents has attracted considerable research attention in

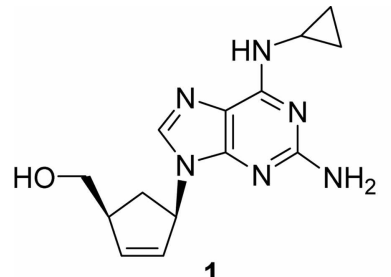

1

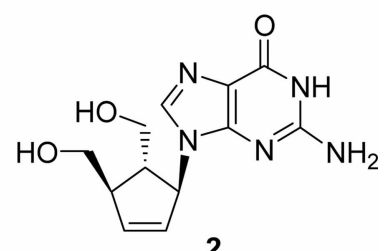

2 the search for novel nucleosides in this class of compound. Recently, several branched nucleosides were synthesized and evaluated as potent antitumor or antiviral agents. Among them, the $4^{\prime} \alpha$-ethenyl compound ${ }^{5}$ and $4^{\prime} \alpha$-ethynyl compound were shown potent antiviral and antitumor activity. Furthermore, $6^{\prime} \alpha$-hydroxymethyl carbovir $2^{7}$ and $6^{\prime} \alpha$-methyl-carbothymidine $3^{8}$ also showed significant antiviral and antitumor activity (Figure 1). Based on these interesting findings of branched nucleosides, a novel class of nucleosides comprising 4 ' $\alpha$-quaternary carbocyclic nucleosides with an additional methyl group at the 6'-position was synthesized.

\section{Results and Discussion}

The quaternary carbon of $\gamma, \delta$-unsaturated ester 5 was constructed successfully using a previously reported procedure." The stereocontrolled introduction of a methyl group in 5 using an ester enolate alkylation (LiHMDS CH3I) provided compounds $\mathbf{6 a}(57 \%)$ and $\mathbf{6 b}(21 \%)$ as diastereomeric mixtures, respectively. Each diastereomer was separated by column chromatography and assigned its stereochemistry by various NMR technique. The relative stereochemical determinations for these compounds would be discussed in the cyclopentenols (10 and $\mathbf{1 1}$ ), which was readily be assigned through the NOE comparison between the proximal protons in the cyclopentenol structures. First, the direct reduction of the ester in toluene solvent at $-78^{\circ} \mathrm{C}$ gave aldehyde 8 in low yield $(36 \%)$. On the other hand, the addition of DIBALH to a solution of the ester 5 in $\mathrm{CH}_{2} \mathrm{Cl}_{2}$ at $0{ }^{\circ} \mathrm{C}$ gave the alcohol derivative 6. which was subjected to oxidation conditions using $\mathrm{PCC}$ to give the aldehyde 8 in a $76 \%$ two step yields.

The addition of vinylmagnesium bromide to the resulting carbonyl compound $\mathbf{8}$ yjelded a bisolefin $\mathbf{9}$ as a diastereomeric mixture, which was not readily separable by conventional column chromatography. The diastereomeric mixture of 9 was not separated but instead subjected to standard ringclosing metathesis conditions using a second-generation Grubbs' catalyst $\left[(\operatorname{lm}) \mathrm{Cl}_{2} \mathrm{PCy}_{3} \mathrm{RuCHP}^{\prime}\right]^{10}$ to predominantly

Figure 1. Antiviral carbonucleosides. 


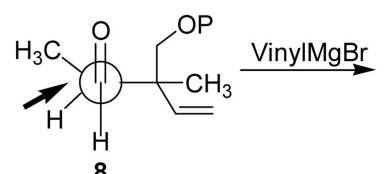

8

Felkin-Anh Model

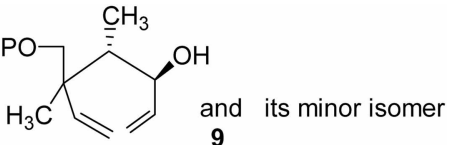

RCM

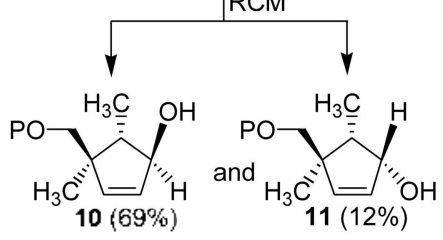

Figure 2. Stercocontrolled addition of nucleophile to aldehyde 8.
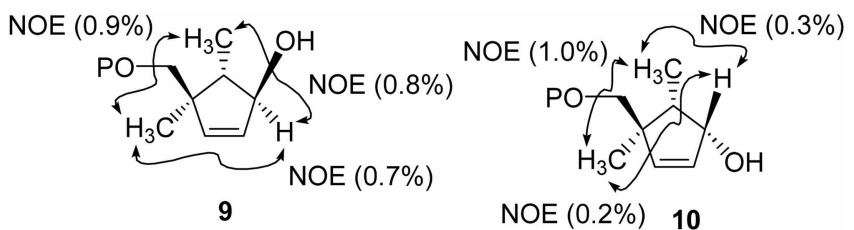

Figur 3. Relative stereochemistry determination hased on NOF. comparisons of compound 9 and 10 .

provide the required cyclopentenol $10(76 \%)$ along with compound $11(11 \%)$ as a minor isomer. The stereochemistry of the cyclized products (10 and 11) was determined by employing the $\mathrm{NOE}$ experiment between the corresponding hydrogen atoms. Upon the irradiation of $\mathrm{C}_{1}-\mathrm{H}$, a relatively strong $\mathrm{NOE}$ was observed at the methyl protons of compound $9\left[\mathrm{C}_{4}-\mathrm{H}(0.7 \%) \& \mathrm{C}_{6}-\mathrm{H}(0.8)\right]$, but not at the methyl protons of compound $10\left[\mathrm{C}_{4}^{-} \mathrm{H}(0.2 \%) \& \mathrm{C}_{1}-\mathrm{H}(0.3)\right]$ (Figure 3). The major stereochemical outcome of compounds 9 and 10 was reasonably explained by a mechanistic rational of the favored $\pi$-facial selection based on the Felkin-Anh rule ${ }^{1]}$ depicted in Figure 2, which shows that the stereochemical assignment of the cyclopentenols $\mathbf{1 0}$ and $\mathbf{1 1}$ was correct. This rule states that the bulkiest of the $\alpha$ ligand (L) is placed in a perpendicular relationship to the plane of the carbonyl group anti to the incoming nucleophile, and the sterically next most bulky $\alpha$ substituent (M) is placed gauche to the carbonyl function. The correct configuration of compound 8 could be assigned based on spectroscopic comparisons observed in compounds $\mathbf{1 0}$ and $\mathbf{1 1}$.

The abacavir analogue was synthesized by activating the cyclopentenol 10 to the ethoxycarbonyl derivative 12 using ethyl chloroformate. Compound $\mathbf{1 2}$ was coupled with the 2amino-6-chloropurine anions generated by $\mathrm{NaH} / \mathrm{DMSO}$ with the [tris(dibenzylodene-acetone)-dipalladium $(0)$ chloroform] ${ }^{12}$ adduct to give the compound 13 (Scheme 2). The required $\beta$-stereochemistry of the nucleosides 13 was controlled successfully from the $\beta$-configuration of compound 10 via a $\mathrm{Pd}(0)$ catalyzed $\pi$-allyl complex mechanism. Compound 13 were desilylated by treating them with tetrabutylammonium fluoride (IBAF) to give the nucleoside 14. Therefore, the exposure of compound 14 to cyclopropylamine in $\mathrm{EtOH}$ under reflux provided the desired nucleoside $\mathbf{1 5 .}$

Based on an extensive literature search, compound 15 appears to be a novel nucleoside. The antiviral evaluations against various viruses such as HIV-I, HSV-I, HSV-2 and HCMV were performed. The synthesized compound 15 showed moderate anti-HIV activity $\left(\mathrm{EC}_{50}=10.67 \mu \mathrm{M}, \mathrm{MT}\right.$ 4 cell lines) $)^{13}$ without any cytotoxicity up to $100 \mu \mathrm{M}$.

In summary, an efficient synthetic method was developed for the synthesis of 4' and 6-dimethylated carbocyclic nucleosides from a simple acetol. This procedure focuses on the simplicity of installing a quaternary carbon and the stereoselectivity in the methylation at cyclopentene ring systems.

\section{Experimental Section}

All chemicals were reagent grade and were used as purchased. All the moisture-sensitive reactions were performed in either a $\mathrm{N}_{2}$ or Ar atmosphere using distilled dry solvents. Elemental analysis was performed using an Elemental Analyzer System (EAll12). The NMR spectra were recorded on a JEOL JNM-LA 300 spectrometer.

(ref)-(2S,3S)-3-(t-Butyldimethylsilyloxymethyl)-3-meth-

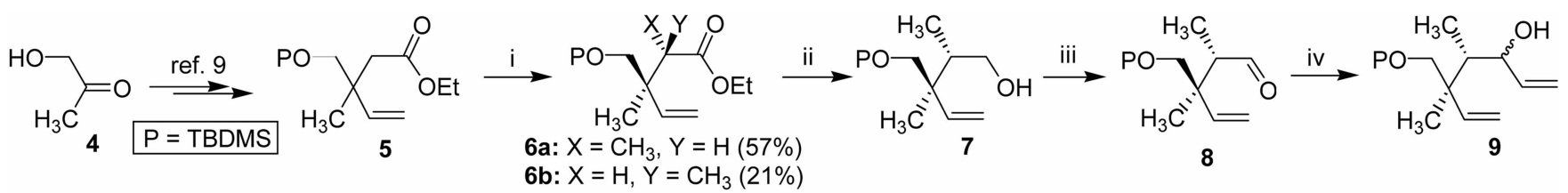

Scheme 1. Synthesis of divinyl intermediate 9. Reaents: i) LiHMDS, $\mathrm{CH}_{3} \mathrm{I}$. THF, $-78^{\circ} \mathrm{C}$ : ii) DIBALH. $\mathrm{CH}_{2} \mathrm{Cl}$. $00^{\circ} \mathrm{C}$ : iv) PCC. 4A MS, $\mathrm{CH}_{2} \mathrm{Cl} \mathrm{I}_{2}+4 \mathrm{~h}$, it: iv) $\mathrm{CH}_{2}=\mathrm{CHMgBr}$. THF.

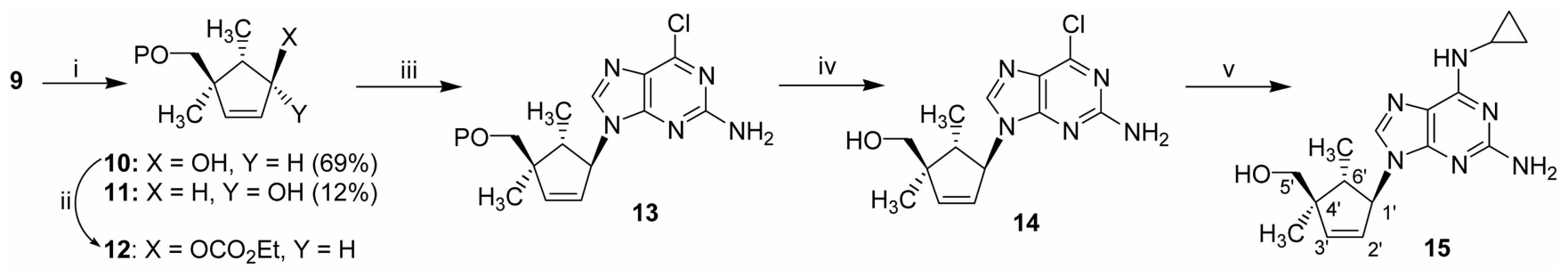

Scheme 2. Synthesis of abacavir analoguc. Reagents: i) 2nd-(jencrution Grubbs' watalyst. $\mathrm{CH}_{2} \mathrm{Cl}_{2}$. reflux. overnight: ii) CICO2Et. DMAP. py ridine. rt, overnight: iii) 2-amino-6-chloropurine. $\mathrm{Pd}_{\mathrm{z}}(\mathrm{dba})_{3} \cdot \mathrm{CHCl}_{i} . \mathrm{P}(\mathrm{O}-\mathrm{i}-\mathrm{Pr})_{3}, \mathrm{VaH}$. THF DMSO, reflux. overnight: iv) TBAF. THF. rt: v) cyclopropylamine. litor I. Rellux. 
yl-2-methyl-pent-t-enoic acid ethyl ester (6a) and (6b): A solution of compound 5 ( $1.23 \mathrm{~g}, 4.3 \mathrm{mmol})$ in tetrahydrofuran $(7 \mathrm{~mL})$ was added to a stirred solution of LiHMDS $(8.6 \mathrm{~mL}, 1.0 \mathrm{M}$ solution in THF) in tetrahydrofuran $(25 \mathrm{~mL})$ using a syringe at $-78^{\circ} \mathrm{C}$. After stirring for $2 \mathrm{hr}$ at the same temperature, the reaction mixture was warmed to $-20^{\circ} \mathrm{C}$ and stirred for an additional $\mathrm{l}$ hr at the same temperature. Methyl iodide $(0.91 \mathrm{~g} .6 .45 \mathrm{~mm}$ ol) was then added to this mixture at $-78{ }^{\circ} \mathrm{C}$ and stirred for $3 \mathrm{~h}$. The mixture was warmed to -25 ${ }^{\circ} \mathrm{C}$ and stirred for an additional $2 \mathrm{~h}$. The reaction was quenched by adding a saturated ammonium chloride solution $(7 \mathrm{~mL})$. The resulting mixture was warmed to room temperature and partitioned between water $(200 \mathrm{~mL})$ and ethyl acetate $(200 \mathrm{~mL})$. The organic layer was dried over anhydrous magnesium sulfate. filtered, concentrated in vacto and purified by colunin chronatography (EtOAc/hexane, $1: 35$ ) to give compounds $6 \mathrm{a}(735 \mathrm{mg} .57 \%$ ) and $6 \mathrm{~b}(271 \mathrm{mg}$. $21 \%$ ): compound for $6 \mathrm{a}:{ }^{1} \mathrm{H} \mathrm{NMR}\left(\mathrm{CDCl}_{3} .300 \mathrm{MHz}\right) \delta 6.02$ (dd. $J=18.6,10.8 \mathrm{~Hz}, 1 \mathrm{H}), 5.08$ (dd. $J=10.8,1.0 \mathrm{~Hz} . \mathrm{lH}$ ). $5.00($ d. $J=18.6 \mathrm{~Hz}, \mathrm{IH}) .4 .04(\mathrm{q} . J=7.2 \mathrm{~Hz} .2 \mathrm{H}), 3.39$ (dd. $J=8.7 .5 .1 \mathrm{~Hz} .2 \mathrm{H}) .2 .7 \mathrm{l}(\mathrm{q}, J=7.4 \mathrm{~Hz} .1 \mathrm{H}), 1.18(\mathrm{t}, J=7.2$ $\mathrm{Hz}, 3 \mathrm{H}), 1.03(\mathrm{~d} . J=7.2 \mathrm{~Hz}, 3 \mathrm{H}) .0 .99(\mathrm{~s}, 3 \mathrm{H}) .0 .88(\mathrm{~s} .9 \mathrm{H})$. $0.02(\mathrm{~s}, 6 \mathrm{H}) ;{ }^{13} \mathrm{C} \mathrm{NMR}\left(\mathrm{CDCl}_{3} .75 \mathrm{MHz}\right) \delta 175.57 .142 .28$. $113.51,68.99,59.86,43.74 .25 .85,18.26 .14 .30 .12 .62$. -5.56; Anal calc for $\mathrm{C}_{16} \mathrm{H}_{3} \mathrm{O}_{3} \mathrm{Si}$ : C. 63.95: $\mathrm{H}, 10.73$ : Found: C. $64.16: \mathrm{H}, 10.60$; compound for $6 \mathrm{~b}:{ }^{1} \mathrm{H}$ NMR $\left(\mathrm{CDCl}_{3} .300\right.$ MHz) $\delta 5.82(\mathrm{dd}, J=18.4 .10 .2 \mathrm{~Hz}, 1 \mathrm{H}), 5.09$ (dd, $J=10.2$. $0.9 \mathrm{~Hz}, \mathrm{IH}$ ). 5.98 (d. $J=18.4 \mathrm{~Hz} . \mathrm{lH}), 4.06$ (q. $J=7.2 \mathrm{~Hz}$. $2 \mathrm{H}) .3 .37(\mathrm{~d}, J=8.4 \mathrm{~Hz} .2 \mathrm{H}) .2 .68(\mathrm{q}, J=7.5 \mathrm{~Hz} . \mathrm{lH}) .1 .18$ (t. $J=7.2 \mathrm{~Hz} .3 \mathrm{H}), 1.02$ (d. $J=7.2 \mathrm{~Hz} .3 \mathrm{H}), 0.97(\mathrm{~s}, 3 \mathrm{H}$ ). $0.87(\mathrm{~s}, 9 \mathrm{H}), 0.02$ (s. $6 \mathrm{H}):{ }^{13} \mathrm{C}$ NMR $\left(\mathrm{CDCl}_{3}, 75 \mathrm{MHz}\right) \delta$ $175.54,142.10,113.74,68.84 .59 .78 .43 .64 .25 .81 .18 .27$. 14.27. 12.34, -5.60: Anal calc for $\mathrm{C}_{16} \mathrm{H}_{3}=\mathrm{O}_{3} \mathrm{Si}: \mathrm{C}, 63.95 ; \mathrm{H}$. 10.73. Found: C. $63.84 ;$ H. 10.80.

(ret)-(2S,3S)-3-(t-Butyldimethylsilyloxymethyl)-3-methyl-2-methyl-pent-t-enol (7): DIBALH $(28.35 \mathrm{~mL}, 1.0 \mathrm{M}$ solution in hexane) was added slowly to a solution of compound $6 \mathrm{a}(4.06 \mathrm{~g} .13 .5 \mathrm{mmol})$ in $\mathrm{CH}_{2} \mathrm{Cl}_{2}(200 \mathrm{~mL})$ at 0 ${ }^{\circ} \mathrm{C}$ and stirred for $3 \mathrm{~h}$ at the same temperature. Methanol (28 $\mathrm{mL}$ ) was then added to the mixture. The resulting mixture was stirred at room temperature for $3 \mathrm{~h}$. and the solid was filtered through a Celite pad. The filtrate was concentrated under vacuum. and the residue was purified by silica gel column chromatography (EtOAc/hexane 1:24) to give compound $7(3.21$ g. $92 \%)$ as a colorless oil: ${ }^{1} \mathrm{H}$ NMR $\left(\mathrm{CDCl}_{3}, 300 \mathrm{MHz}\right) \delta 5.81(\mathrm{dd} . J=18.8,10.4 \mathrm{~Hz}, \mathrm{LH}), 5.01$ $($ d. $J=18.8 \mathrm{~Hz} . \mathrm{lH}), 4.89(\mathrm{~d}, J=18.2 \mathrm{~Hz} \cdot \mathrm{lH}), 3.42(\mathrm{dd} . J=$ $13.5 .2 .4 \mathrm{~Hz}, 1 \mathrm{H}) .3 .32$ (dd. $J=9.9 .5 .4 \mathrm{~Hz} .1 \mathrm{H}) .1 .72(\mathrm{q} . J=$ $6.8 \mathrm{~Hz}, \mathrm{lH}), 0.90(\mathrm{~s} .3 \mathrm{H}), 0.88(\mathrm{~d} J=3.3 \mathrm{~Hz}, 3 \mathrm{H}), 0.84$ (s. 9H) 0.02 (s. $6 \mathrm{H}):{ }^{13} \mathrm{C}$ NMR $\left(\mathrm{CDCl}_{3 .} 75 \mathrm{MHz}\right) \delta 1+3.61$. $113.00,68.86,64.72,43.46 .41 .14,25.64$. 18.20. 17.18. 12.29. -5.62: Anal calc for $\mathrm{C}_{14} \mathrm{H}_{33} \mathrm{O}_{-} \mathrm{Si}$. C. $65.06 ; \mathrm{H} .11 .70$. Found: C. $64.89 ;$ H. 11.58

(rel)-(2S,3S)-3-(t-Butyldimethylsilyloxymethyl)-3-methyl-2-methyl-pent-4-enal (8): $4 \AA$ A molecular sieves (11.0 g) and PCC $(9.0 \mathrm{~g} .42 \mathrm{mmol})$ were added slowly to a solution of compound $7(3.77 \mathrm{~g} .14 .58 \mathrm{mmol})$ in $\mathrm{CH}_{2} \mathrm{Cl}_{2}(110 \mathrm{~mL})$, at
$0{ }^{\circ} \mathrm{C}$. and stirred ovemight at room temperature. Excess diethyl ether $(400 \mathrm{~mL})$ was then added. The mixture was stirred vigorously for $4 \mathrm{~h}$ at the same temperature, and the resulting solid was filtered through a short silica gel column. The filtrate was concentrated under vacuum and the residue was purified by silica gel column chromatography (EtOAc/ hexane, 1:30) to give compound $8(3.21 \mathrm{~g} .86 \%)$ as a colorless oil: ${ }^{1} \mathrm{H}$ NMR $\left(\mathrm{CDCl}_{3} .300 \mathrm{MHz}\right) \delta 9.72$ (s. $\mathrm{HH}$ ), $5.95($ dd $, J=17.7 .11 .1 \mathrm{~Hz}, \mathrm{lH}) .5 .13(\mathrm{~d} . J=11.2 \mathrm{~Hz} .1 \mathrm{H})$, $5.08(\mathrm{~d}, J=17.7 \mathrm{~Hz}, 1 \mathrm{H}), 3.48(\mathrm{dd} . J=9.9 .4 .8 \mathrm{~Hz}, 2 \mathrm{H}), 2.48$ (q. $J=6.8 \mathrm{~Hz}, 1 \mathrm{H}), 1.00(\mathrm{~d}, J=7.5 \mathrm{~Hz}, 3 \mathrm{H}), 0.98$ (s. $3 \mathrm{H})$, 0.86 (s. 9H), 0.02 (s. $6 \mathrm{H}$ ); ${ }^{12} \mathrm{C} \mathrm{NMR}\left(\mathrm{CDCl}_{3} .75 \mathrm{MHz}\right) \delta$ $205.04,141.85 .114 .25,68.58,50.75,44.18,25.77,18.49$. 18.20. 8.59. -5.68 .

$(r e t)-(3 R$ and $3 S, 4 S, 5 S)-5-(t-B u t y l d i m e t h y l s i l y l o x y-$ methyl)-5-methyl-4-methyl-hepta-1,6-dien-3-ol (9): Vinyl magnesium bromide ( $16.9 \mathrm{~mL}, 1.0 \mathrm{M}$ solution in THF) was added slowly to a solution of compound 8 (3.62 g. 14.13 mumol) in dry THF $(150 \mathrm{~mL})$ at $-78^{\circ} \mathrm{C}$. After $2 \mathrm{~h}$. a saturated $\mathrm{NH}_{4} \mathrm{Cl}$ solution $(14 \mathrm{~mL})$ was added, and the reaction mixture was warmed slowly to room temperature. The mixture was extracted with EtOAc $(2 \times 300 \mathrm{~mL})$. The combined organic layer was dried over $\mathrm{MgSO}_{4}$. filtered. and evaporated. The residue was purified by silica gel column chromatograply (EtOAc/hexane. 1:25) to give the divinyl 9 ( $3.42 \mathrm{~g} .85 \%$ ) as a diastereomeric mixture: ${ }^{1} \mathrm{H}$ NMR $\left(\mathrm{CDCl}_{3}\right.$, $300 \mathrm{MHz}) \delta 5.92(\mathrm{dd}, J=18.1 .10 .8 \mathrm{~Hz} .1 \mathrm{H}) \cdot 5.75-5.69(\mathrm{~m}$, lH). 5.04-4.83 (m. 2H). 3.59-3.27 (m. 3H), 1.54 (m. lH), $1.02(\mathrm{~s}, 3 \mathrm{H}) .0 .9 \mathrm{l}$ (s. 3H). 0.82-0.79 (m, 12H), $0.02(\mathrm{~s}, 6 \mathrm{H})$ : ${ }^{13} \mathrm{C}$ NMR $\left(\mathrm{CDCl}_{3} .75 \mathrm{MHz}\right) \delta 144.82 .143 .94$. 141.32, $141.18,113.53,112.44 .71 .31 .70 .80 .67 .84,67.28 .45 .84$. $45.11,44.12 .21 .33,20.64$. 18.34, 7.13, -5.61 ; Anal calc for $\mathrm{C}_{16} \mathrm{H}_{32} \mathrm{O}_{2} \mathrm{Si}: \mathrm{C}, 67.54: \mathrm{H}, 11.34$. Found: $\mathrm{C}, 67.42: \mathrm{H}, 11.25$.

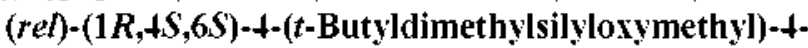
methyl-5-methyl-cyclopent-2-enol (10) and (rel)-(1S,+S, 6S)-4-(t-Butyldimethylsilyloxymethyl)-4-methyl-5-methyl-cyclopent-2-enol (11): A $2^{\text {nd }}$ generation Grubbs' catalyst ( $80 \mathrm{mg} .0 .11 \mathrm{mmol}$ ) was added to a solution of compound 11 (3.3 g. $11.68 \mathrm{mmol})$ in dry $\mathrm{CH}_{2} \mathrm{Cl}_{2}(40 \mathrm{~mL}$ ). The reaction mixture was refluxed overnight. and concentrated. The residue was purified by silica gel column chromatography (EtOAc/hexane. 1:25) to give the compounds $10(2.1 \mathrm{~g}$ $69 \%)$ and 11 (359 $\mathrm{mg} .12 \%)$ as colorless oils: compound 10 . ${ }^{\mathrm{l}} \mathrm{H} \mathrm{NMR}\left(\mathrm{CDCl}_{3}, 300 \mathrm{MHz}\right) \delta 5.82(\mathrm{~d} . J=5.8 \mathrm{~Hz}, \mathrm{lH}) .5 .56$ (d. $J=5.9 \mathrm{~Hz} .1 \mathrm{H}), 4.49(\mathrm{~d}, J=6.5 \mathrm{~Hz}, 1 \mathrm{H}) .3 .48(\mathrm{~d} . J=9.9$ Hz. $1 \mathrm{H}$ ). 3.28 (d. $J=9.9 \mathrm{~Hz}, 1 \mathrm{H}$ ). 1.57 (q. $J=7.5 \mathrm{~Hz} .1 \mathrm{H}$ ), 1.14 (d. $J=7.2 \mathrm{~Hz}, 3 \mathrm{H}$ ), 0.98 (s. $3 \mathrm{H}$ ). 0.85 (s. $9 \mathrm{H}$ ). 0.02 (s. $6 \mathrm{H}):{ }^{13} \mathrm{C}$ NMR $\left(\mathrm{CDCl}_{3}, 75 \mathrm{MHz}\right) \delta 139.84 .134 .18,84.10$, 67.05. 53.46, 51.56. 25.95. 22.72, 18.11, 10.63. -5.57: Anal calc for $\mathrm{C}_{14} \mathrm{H}_{28} \mathrm{O}_{2} \mathrm{Si}$ : C. 65.57: H, 11.00. Found: $\mathrm{C}, 65.47 \mathrm{H}$. 10.83; compound 11: ${ }^{1} \mathrm{H}$ NMR $\left(\mathrm{CDCl}_{3,3} 300 \mathrm{MHz}\right) \delta 5.80(\mathrm{~d}$, $J=6.0 \mathrm{~Hz} . \mathrm{lH}), 5.59(\mathrm{~d}, J=6.0 \mathrm{~Hz}, \mathrm{lH}) .4 .50(\mathrm{~d} . J=6.6 \mathrm{~Hz}$, $1 \mathrm{H}) .3 .45(\mathrm{~d} . J=9.8 \mathrm{~Hz} .1 \mathrm{H}), 3.26(\mathrm{~d} . J=9.8 \mathrm{~Hz}, 1 \mathrm{H}) .1 .55$ (q. $J=7.4 \mathrm{~Hz}, 1 \mathrm{H}$ ), 1.16 (d. $J=7.3 \mathrm{~Hz}, 3 \mathrm{H}), 0.97$ (s. $3 \mathrm{H}$ ), 0.85 (s. 9H), 0.02 (s. $6 \mathrm{H}$ ): ${ }^{12} \mathrm{C} \mathrm{NMR}\left(\mathrm{CDCl}_{3} .75 \mathrm{MHz}\right) \delta$ $140.12,134.32,83.98,66.94,53.32,51.12,25.62 .22 .67$. 18.49. 10.28. -5.53; Anal calc for $\mathrm{C}_{14} \mathrm{H}_{28} \mathrm{O} 32 \mathrm{Si}$ C 65.57 : $\mathrm{H}$, 
11.00. Found: C. $65.68 ;$ H. 11.17

(ret)-(1R,4S,6S)-1-Ethoxycarbonyloxy-4-( $t$-butyldimethylsilyloxymethyl)-4-methyl-5-methyl-cyclopent-2-ene (12): Ethyl chloroformate ( $1.44 \mathrm{~mL}, 10.1 \mathrm{mmol}$ ) and DMAP (100 mg. $0.72 \mathrm{~mm}$ mol) were added to a solution of compound $\mathbf{1 0}$ $(2.4$ g. $9.36 \mathrm{~nm}$ ol) in anhydrous pyridine $(30 \mathrm{~mL})$. The reaction mixture was stirred overnight at room temperature. The reaction was quenched using a saturated $\mathrm{NaHCO}_{3}$ solution $(2.5 \mathrm{~mL})$ and concentrated under reduced pressure. The residue was extracted with EtOAc, dried over $\mathrm{MgSO}_{4}$. filtered, and concentrated. The residue was purified by silica gel column chronatography (EtOAc/hexane, 1:25) to give contpound $12(2.43 \mathrm{~g} .79 \%)$ as a colorless syrup: ${ }^{1} \mathrm{H}$ NMR $\left(\mathrm{CDCl}_{3}, 300 \mathrm{MHz}\right) \delta 5.83$ (dd. $\left.J=5.4 .1 .4 \mathrm{~Hz}, 1 \mathrm{H}\right), 5.72$ (dd. $J=5.4 .1 .8 \mathrm{~Hz}, \mathrm{IH}) .5 .21(\mathrm{dt}, J=6.6 .1 .5 \mathrm{~Hz}, \mathrm{IH}) .4 .19(\mathrm{q}, J$ $=7.2 \mathrm{~Hz}, 2 \mathrm{H}$ ), 3.37 (s. $2 \mathrm{H}$ ). 2.06 (quint. $J=7.5 \mathrm{~Hz} . \mathrm{IH}$ ). 1.31 (t. $J=7.2 \mathrm{~Hz}, 3 \mathrm{H}$ ). 1.02 (d. $J=7.2 \mathrm{~Hz} .3 \mathrm{H}), 0.90(\mathrm{~s}$. $3 \mathrm{H}), 0.86(\mathrm{~s}, 9 \mathrm{H}), 0.02(\mathrm{~s}, 6 \mathrm{H}):{ }^{13} \mathrm{C} \mathrm{NMR}\left(\mathrm{CDCl}_{3} .75 \mathrm{MHz}\right)$ $\delta 155.30 .143 .39 .127 .96 .89 .87,89.46,70.44,63.76,51.28$, 43.88. 25.86, 18.15, 14.26. 13.01. 10.76. -5.54: Anal calc for $\mathrm{C}_{17} \mathrm{H}_{32} \mathrm{O}_{4} \mathrm{Si}: \mathrm{C}, 62.15: \mathrm{H}, 9.82$. Found: C. 62.05: H, 9.95.

(rel)-(1'R,4'S,6'S)-9-[+-(t-Butyldimethylsilyloxymethy])4-methyl-6-methyl-cyclopent-2-en-1-yl] 2-amino-6chloropurine (13): 2-Amino-6-chloropurine (383 mg. 2.26 mmol) was added to pure $\mathrm{NaH}(57.6 \mathrm{mg}$. $2.4 \mathrm{munol})$ in anhydrous DMSO $(9.0 \mathrm{~mL})$. The reaction mixture was stirred for $40 \mathrm{~min}$ at $50-60{ }^{\circ} \mathrm{C}$ and then cooled to room temperature. For the preparation of the catalytic solution. $\mathrm{P}(\mathrm{O}-\mathrm{i}-\mathrm{Pr})_{3}(10.08 \mathrm{~mL} .2 .26 \mathrm{mmol})$ was added to a solution of $\mathrm{Pd}_{2}(\mathrm{dba})_{3}, \mathrm{CHCl}_{3}(53.12 \mathrm{mg}, 28.8 \mathrm{mmol})$ in anhydrous THF $(15.0 \mathrm{~mL})$, which was then stirred for $40 \mathrm{~min}$. A cataly st solution of THF and formate starting material 12 (657 $\mathrm{mg}$. $2.0 \mathrm{mumol})$ dissolved in anlydrous THF (15 mL) was then added slowly to the reaction mixture. The reaction mixture was stirred overnight under reflux and then quenched with water $(7 \mathrm{~mL})$. The reaction solvent was removed under reduced pressure. The residue was purified by silica gel column chromatography $\left(\mathrm{MeOH} / \mathrm{CH}_{2} \mathrm{Cl}_{2}, 1: 10\right)$ to give compound 13 (261 mg. $32 \%$ ): ${ }^{1} \mathrm{H} \mathrm{NMR} \mathrm{(CDCl}, 300 \mathrm{MHz}$ ) $\delta 7.95$ (s. IH). $5.82(\mathrm{dd}, J=5.8,1.8 \mathrm{~Hz}, \mathrm{IH}) .5 .69(\mathrm{dd}, J=$ $6.0 .1 .8 \mathrm{~Hz}, 1 \mathrm{H}), 5.39(\mathrm{dd} J=11.4 .2 .0 \mathrm{~Hz}, 1 \mathrm{H}), 3.38(\mathrm{dd} . J$ $=9.9 .2 .1 \mathrm{~Hz}, 2 \mathrm{H}) .2 .29$ (quint $J=7.8 \mathrm{~Hz}, 1 \mathrm{H}) .1 .01(\mathrm{~d} . J=$ $7.6 \mathrm{~Hz} .3 \mathrm{H}), 0.90$ (s. $3 \mathrm{H}), 0.86(\mathrm{~s} .9 \mathrm{H}) .0 .02$ (s. $6 \mathrm{H}){ }^{13} \mathrm{C}$ NMR $\left(\mathrm{CDCl}_{3} .75 \mathrm{MHz}\right) \delta$ 159.20. 154.27, 151.21. 143.11. 140.54, 134.67. 125.31, 68.81, 65.79. 52.91, 47.31. 25.71. 21.57, 18.22, 12.23, 10.93, -5.57: Anal calc for $\mathrm{C}_{19} \mathrm{H}_{3(1 \mathrm{CIN}} \mathrm{CSSi}_{\mathrm{O}} \mathrm{C}$. 55.93; H. 7.41; N, 17.16. Found: C. 56.13: H. 7.55; N. 17.01.

(rel)-(1'R,4'S,6'S)-9-[4-(Hydroxymethyl)-4-methyl-6methyl-cyclopent-2-en-1-yl] 2-amino-6-chloropurine (14): TBAF $\left(0.57 \mathrm{~mL}, 1.0 \mathrm{M}\right.$ solution in THF) at $0{ }^{\circ} \mathrm{C}$ was added to a solution of compound 13 (111.6 $\mathrm{mg}, 0.38 \mathrm{mmol}$ ) in THF $(10 \mathrm{~mL}$ ). The mixture was stirred overnight at room temperature and concentrated. The residue was purified by silica gel columu chromatography $\left(\mathrm{MeOH} / \mathrm{CH}_{2} \mathrm{Cl}_{2} .15\right)$ to give compound $14(79.25 \mathrm{mg}, 71 \%)$ as a white solid: $\mathrm{mp}$ 178-180 ${ }^{\circ} \mathrm{C}:{ }^{1} \mathrm{H}$ NMR (DMSO-d. $\left.300 \mathrm{MHz}\right) \delta 7.95$ (s. IH). 5.81 (dd. $J=5.4 .2 .1 \mathrm{~Hz} .1 \mathrm{H}), 5.57(\mathrm{dd}, J=6.0,1.5 \mathrm{~Hz}, \mathrm{IH})$. $5.07(\mathrm{dt}, J=8.4,1.8 \mathrm{~Hz}, 1 \mathrm{H}) .4 .87(\mathrm{t}, J=5.4 \mathrm{~Hz} .1 \mathrm{H}) .3 .29$ (d. $J=10.5 \mathrm{~Hz} .1 \mathrm{H}$ ). 3.12 (d. $J=10.5 \mathrm{~Hz}, \mathrm{lH}$ ). 1.98 (quint. $J$ $=8.1 \mathrm{~Hz} . \mathrm{lH}$ ). 0.98 (d, $J=7.2 \mathrm{~Hz} .3 \mathrm{H}) .0 .89$ (s. $3 \mathrm{H}) ;{ }^{13} \mathrm{C}$ NMR (DMSO- $\left.c_{i}, 75 \mathrm{MHz}\right) \delta$ 159.76. 154.81. 150.99. 142.84, 140.85, 135.01. 125.67.65. 65.42. 51.53, 40.33 . 17.52. 12.47: Anal calc for $\mathrm{C}_{13} \mathrm{H}_{16} \mathrm{ClN}_{5} \mathrm{O}: \mathrm{C}, 53.15 ; \mathrm{H} .5 .49$. N, 23.84. Found: C, 53.27: H, 5.55; N. 23.73.

(rel)-(1'R,4'S,6'S)-9-[4-(Hydroxymethyl)-4-methyl-6methyl-cyclopent-2-en-1-yl] 2-amino-6-cyclopropylpurine (15): Cyclopropyl amine (0.114 mL. 1.65 mmol) was added to a solution of compound $14(96.9 \mathrm{mg} .0 .33 \mathrm{mmol})$ in $\mathrm{EtOH}$ $(12 \mathrm{~mL})$ and refluxed for $5 \mathrm{~h}$. After cooling. the reaction mixture was concentrated under reduced pressure. The residue was purified by silica gel column chromatography ( $\mathrm{MeOH} / \mathrm{CH}_{2} \mathrm{Cl}_{2}, \mathrm{l}: 5$ ) to give compound 15 (62 $\mathrm{mg} .68 \%$ ) as a solid: mp 181-183: ${ }^{1} \mathrm{H}$ NMR (DMSO- $\left.d_{6,}, 300 \mathrm{MHz}\right) \delta 7.97$ (s. $\mathrm{lH}$ ). 5.80 (dd, $J=5.6 .2 .0 \mathrm{~Hz}, \mathrm{lH}), 5.65$ (dd, $J=6.2 .2 .0$ Hz, IH). 5.24 (dt. $J=8.2,1.8 \mathrm{~Hz}, 1 \mathrm{H}) .4 .89$ (t. $J=5.2 \mathrm{~Hz}$, $\mathrm{lH}), 3.02(\mathrm{~m} . \mathrm{lH}), 2.37(\mathrm{~d} . J=10.2 \mathrm{~Hz}, \mathrm{lH}) .2 .29(\mathrm{~d} . J=10.5$ $\mathrm{Hz}, \mathrm{lH}), 2.04$ (quint $J=8.0 \mathrm{~Hz} . \mathrm{H}) .0 .99(\mathrm{~d}, J=7.6 \mathrm{~Hz}, 3 \mathrm{H})$, $0.92(\mathrm{~s}, 3 \mathrm{H}), 0.57-0.71(\mathrm{~m}, 4 \mathrm{H}) ;{ }^{12} \mathrm{C}$ NMR (DMSO- $c_{6}, 75$ $\mathrm{MHz}) \delta 158.98 .153 .79,150.21 .142 .23 .141 .47,134.87$, $125.14,67.66 .64 .18 .50 .39,41.32,23.79$. 16.99, 12.71. 6.43; Anal calc for $\mathrm{C}_{16 \mathrm{H}_{2}} \mathrm{~N}_{6} \mathrm{O}: \mathrm{C}, 61.13 ; \mathrm{H}, 7.05 ; \mathrm{N}, 26.73$. Found: C. 60.90 : H. 6.92; N, 26.68; MS (EI) $\mathrm{n} / \mathrm{z} 315(\mathrm{M}+1)^{+}$.

Acknowledgment. This study was supported by research grant of Chosun University, 2007.

\section{References}

1. (a) Agrofoglio. L: Suhas, E: Farese, A.: Condom, R.: Challand. S.; Earl. R. A. Tetraledron 1994, 50. 10611. (b) Borthwick. A. D: Biggadike. K. Tetrahedon 1992. 48.571.

2. (a) Herdewijn. P.: De Clercq. E.: Balzarini. T.: Vanderhaeghe. H. $J$ Med Chem. 1985. 28.550. (b) Palmer. J. L.: Abeles. R. H. J. Biol Chem. 1979, 254. 1217. (c) Ueland. P. 1. Pharmacol. Rev 1982. 34.223.

3. Daluge, S. M.: Good. S. S.; Faletto, M. B.; Miller. W. H.; St Clair. M. H.: Boone. L. R.: Tisdale. M.: Parry. N. R.: Reardon. J. E.: Dornsife. R. E.: Averett. D. R.: Krenitsky. T. A. Antinicrob. Agents Chentother. 1997. H1. 1082 .

4. Levine, S.; Hernandez. D.: Yamanaka. G.: Zhang. S.; Rose, R: Weinheimer, S.; Colonno. R. J. Antwicrob. Agents Chemother. 2002. 16,2525

5. Sugimoto. I.: Shuto. S.: Mori. S.: Shigeta. S.: Matuda. A. Bioorg. Med. Chem. Lett. 1999. 9. 285.

6. Ohrui. H: Kohgo. S.: Kitano. K.: Sakata. S.: Kodama. E.: Yoshimura. K.: Matsuoka. M.: Shigeta. S.: Mitsuya. H. H. J. I led. Chem. 2000, 43,4516 .

7. Katagiri, N.; Nomura, M.: Sato. H.: Kaneko. C.: Yusa. K.; Tsuruo. T. J. Med Chem. 1992. 35. 1882

8. Tanaka. M.: Norimine. Y.: Fujita. T.: Suemune. H.: Sakai. K. J. Org. Chen 1996.61.6952

9. Hong. J. H.: Ko. O. H. Bull Koran Chem. Soc. 2003. 24. 1284.

10. (a) Fürster, A. Angew. Chem. Int. Ed 2003, 39. 3012. (b) McReynolds, M. D.: Doughert: J. M.: Hanson, P. R. Chem. Rev 2004. 104. 2239.

11. Mengel. A.: Reiser. O. Chent. Rev 1999.99. 1191.

12. Crimmins. M. T.: King. B. W.: Zuercher. W. I:: Choy. A. L. J. Org. Chem. 2000. 65, 8499

13. Pauwels, R: Balzarini. J.: Baba, M; Snoeck. R.: Schols, D; Herdewijn, P. J. Irol l hethods 1988. 20. 309. 